\title{
Simone Waldt and Klaus Woertler (Eds): Measurements and Classifications in Musculoskeletal Radiology
}

\author{
Georg Thieme Verlag, Stuttgart, 2014; ISBN 978-3-13169271-9
}

\author{
Giuseppe D. Di Stasio • Luigi Mansi
}

Published online: 27 May 2014

(C) Springer-Verlag Berlin Heidelberg 2014

Measurements and Classifications in Musculoskeletal Radiology is the authorized translation of the first German edition, published in 2011 by Georg Thieme. The editors are Simone Waldt and Klaus Woertler, respectively associate professor and professor of radiology at the Technische Universitat München (TUM) in Munich. The contribution of Matthias Eiber is also recognized.

This is a very well structured book of 214 pages, enriched with 423 illustrations, including images, schema and tables, in which students, residents and experienced doctors can find valuable help and an important reference. Across 17 chapters, the publication guides the reader through the main topics of musculoskeletal imaging, analyzing a variety of measurement schemes and classifications commonly used in orthopedics (excluding fractures). It covers lower limb alignment, all major joints (hip, knee, foot, shoulder, elbow, wrist and hand) as well as the spine and cranio-cervical junction, grading/ staging of bone and soft tissue tumors, osteoporosis, articular cartilage lesions, rheumatoid arthritis, hemophilia, muscle injuries and skeletal age. In order to be as accurate as possible, the authors have always tried to refer to the original primary sources, which are necessarily dated but nonetheless relevant.
According to this approach, all outmoded practices and systems were excluded from this collection or were included with appropriate comments noting at the same time the actual, practical value of specific methods. This "handy reference for everyday use" manages to gather all the currently fragmented information on musculoskeletal radiology measurements and classification systems into one text, full of detailed illustrations and radiological images where reference lines, markings, angles and key values help the reader to focus on the main concepts and get directly to the point. Moreover, throughout the text are gray boxes marked with an exclamation mark in which key points, clinical pearls and pitfall alerts are usefully highlighted. This kind of layout improves the overall quality of the book, allowing it to provide both a more-than-sufficient level of detail and a positive conciseness.

From this perspective, Measurements and Classifications in Musculoskeletal Radiology does not replace a textbook but can be rightfully considered an "external memory" to always have nearby, perfect to deal with the endless measurement techniques and classification systems used in orthopedic imaging and the ideal companion for use at the radiology workstation.
G. D. Di Stasio $\cdot$ L. Mansi $(\bowtie)$

Second University of Naples, Naples, Italy

e-mail: luigi.mansi@unina2.it 